\title{
Mineração
}

\section{Controle de qualidade no processamento de polpas de caulim utilizando propriedades óticas}

\author{
James José Varela \\ Pesquisador MSc, Programa de Pós-Graduação em Engenharia de Minas \\ Metalúrgica e dos Materiais - Univ. Federal do Rio Grande do Sul.E-mail: varela@ct.ufrgs.br \\ Roberto Gliese \\ Pesquisador PhD, Programa de Pós Graduação em Engenharia de Minas \\ Metalúrgica e dos Materiais - Univ. Federal do Rio Grande do Sul.E-mail: gliese@ct.ufrgs.br \\ Carlos Otávio Petter \\ Professor PhD, Departamento de Engenharia de Minas - Univ. Federal do Rio Grande do Sul \\ E-mail:cpetter@vortex.ufrgs.br \\ Carlos Arthur Peixoto \\ Engenheiro Químico do Núcleo de Tecnologia de Produção - RENNER-HERRMANN \\ E-mail: c.arthur@renner.com.br
}

\section{Resumo}

O caulim é um mineral industrial que necessita um controle de qualidade muito rigoroso, como, por exemplo, o tamanho das partículas e a alvura. Por outro lado, há uma carência na indústria mineral de equipamentos capazes de medir suas propriedades físicas em tempo real.

Nesse trabalho foram avaliou-se o alvejamento químico de uma amostra de caulim em polpa. Foram realizados três ensaios distintos, no laboratório da UFRGS, sendo que os resultados foram comparados com a mesma amostra alvejada em escala industrial. Atualmente o processamento de caulim é avaliado através do espectro de reflectância determinado em pastilhas confeccionadas a partir de amostras secas desse minério. Essa forma de análise, além de estar sujeita a erros de amostragem, preparação e medição da amostra, demanda muito tempo. O sistema utilizado nesse trabalho para caracterização on-line de polpas minerais é uma versão adaptada do equipamento Colorcell original. Esse equipamento foi desenvolvido pela empresa RENNER-HERRMANN para a produção de tintas.

Observou-se uma boa correlação linear dos dados de alvura e amarelamento obtidos através dos dois métodos de caracterização óptica. Os resultados desse trabalho possuem um grande potencial de aplicação na indústria, principalmente, para reduzir custos operacionais e obter produtos finais de qualidade através do controle on-line.

\begin{abstract}
Kaolin is an industrial mineral that needs a very rigorous quality control, such as fine particles size and high brightness, to meet market quality. On the other hand, in the mineral industry, there is a lack of equipment capable of evaluating processes in actual time.

In this work, chemical bleaching of one kaolin sample, in pulp, was evaluated. Three different tests were performed at the UFRGS laboratory and the results were compared to one industrially bleached sample. At present, the control of kaolin processing is accomplished by reflectance spectrum analysis using tablets prepared from dry ore samples. This type of analysis, besides being error sensitive in sample preparation and measurement, is very time consuming. The apparatus used in this work, for on-line characterization of mineral pulps, is an adapted version of the original Colorcell ${ }^{\mathbb{E}}$ equipment. Colorcell ${ }^{\mathbb{R}}$, developed by the RENNER-HERRMANN Company, is used in the continuous production process of paints.

A good linear data correlation was observed between the dry and wet optical methods of characterization. The results of this work possess a great application potential in industry, mainly, to reduce operational costs and improve quality of the final product.
\end{abstract}

Keywords: Kaolin, process control, Colorcell, whiteness.

Palavras-chave: Caulim, controle de processo, Colorcell, alvura. 


\section{Introdução}

O Brasil e os EUA possuem as maiores reservas mundiais de caulim. Os Estados do Pará e do Amapá são os maiores produtores nacionais, contribuindo com mais de $90 \%$ desse mineral industrial. As reservas de caulim podem ser divididas nos seguinte dois grupos, segundo a sua gênese: caulim primário e caulim secundário. São as características de cada depósito que irão determinar as etapas de beneficiamento necessárias para a sua produção. A seguir serão detalhadas as etapas de beneficiamento necessárias para a produção de caulim em uma empresa localizada na região sul do Brasil (Varela, 2002). Em primeiro lugar, os distintos tipos de caulins ROM (Run Of Mine) provenientes da mina chegam em um pátio e são separados de acordo com a sua qualidade. Com o objetivo de maximizar o aproveitamento do minério e gerar um produto especificado pela empresa, a alimentação da usina é realizada através de misturas (blendings) desses minérios ROM.

A primeira etapa do processamento é a dispersão do minério, concomitantemente com a adição de água. A polpa é, então, direcionada para a etapa de desareiamento/fracionamento por meio de calhas. A etapa de desareiamento/fracionamento na empresa é realizada através de baterias de ciclones e também por uma centrífuga. A polpa, nessa etapa, possui, aproximadamente, $10 \%$ de sólidos. O rejeito dessa etapa (fração compreendida entre 20 e $44 \mu \mathrm{m}$ ) é comercializado como um subproduto para a indústria da borracha. A fração arenosa $(>44 \mu \mathrm{m})$ retorna para a cava da mina. $\mathrm{O}$ produto da etapa desareiamento/fracionamento contendo o material mais fino $(<20 \mu \mathrm{m})$ é espessado para $27 \%$ de sólidos.

A polpa espessada é direcionada para tanques com capacidade de $23,4 \mathrm{~m}^{3}$, onde será realizada a etapa de alvejamento. Essa etapa tem o objetivo de retirar as impurezas dos caulins prejudiciais à sua alvura através de uma lixiviação redutora dos óxidos. A etapa seguinte compreende a filtragem do caulim alve- jado e o seu objetivo é eliminar o ferro solúvel em água e reduzir a umidade do produto.

A última etapa do processamento compreende a secagem do caulim. $\mathrm{Na}$ Figura 1, está representado o fluxograma generalizado do processamento de caulim dessa empresa brasileira. Existem outros processos utilizados para a adequação do caulim às exigências do mercado consumidor, entre os quais podemos citar a delaminação e a separação magnética.

A etapa de alvejamento do caulim, além de determinar a qualidade do produto, também influencia, diretamente, no custo de processamento desse produto. Considerando que ambos os fatores, qualidade e preço do produto, são essenciais para a sua permanência no mercado, é de extrema importância a correta compreensão e otimização da etapa de alvejamento do caulim. Nesse trabalho, o equipamento Colorcell foi utilizado com o objetivo de medir as diferenças de cor resultantes da etapa de alvejamento.

\section{Materiais e métodos}

\subsection{Amostras}

As amostras de caulim utilizadas nesse trabalho foram coletadas em uma usina de processamento de caulim localizada no município de Pântano GrandeRS. O local de amostragem foi a etapa de alvejamento. A amostra 1 representa a alimentação da etapa de alvejamento e a amostra 2 corresponde ao produto da etapa de alvejamento. As amostras I, II e III representam os alvejamentos químicos realizados no laboratório da UFRGS.

\subsection{Caracterização colorimétrica}

Sistemas colorimétricos são utilizados em aplicações de controle de qualidade dos produtos, quando há a necessidade de se quantificarem cores. O sistema colorimétrico mais amplamente utilizado e recomendado pelo CIE (International Commission on Illumination) é o espaço uniforme CIELAB 1976 (CIEL*a*b*), ver Figura 2.

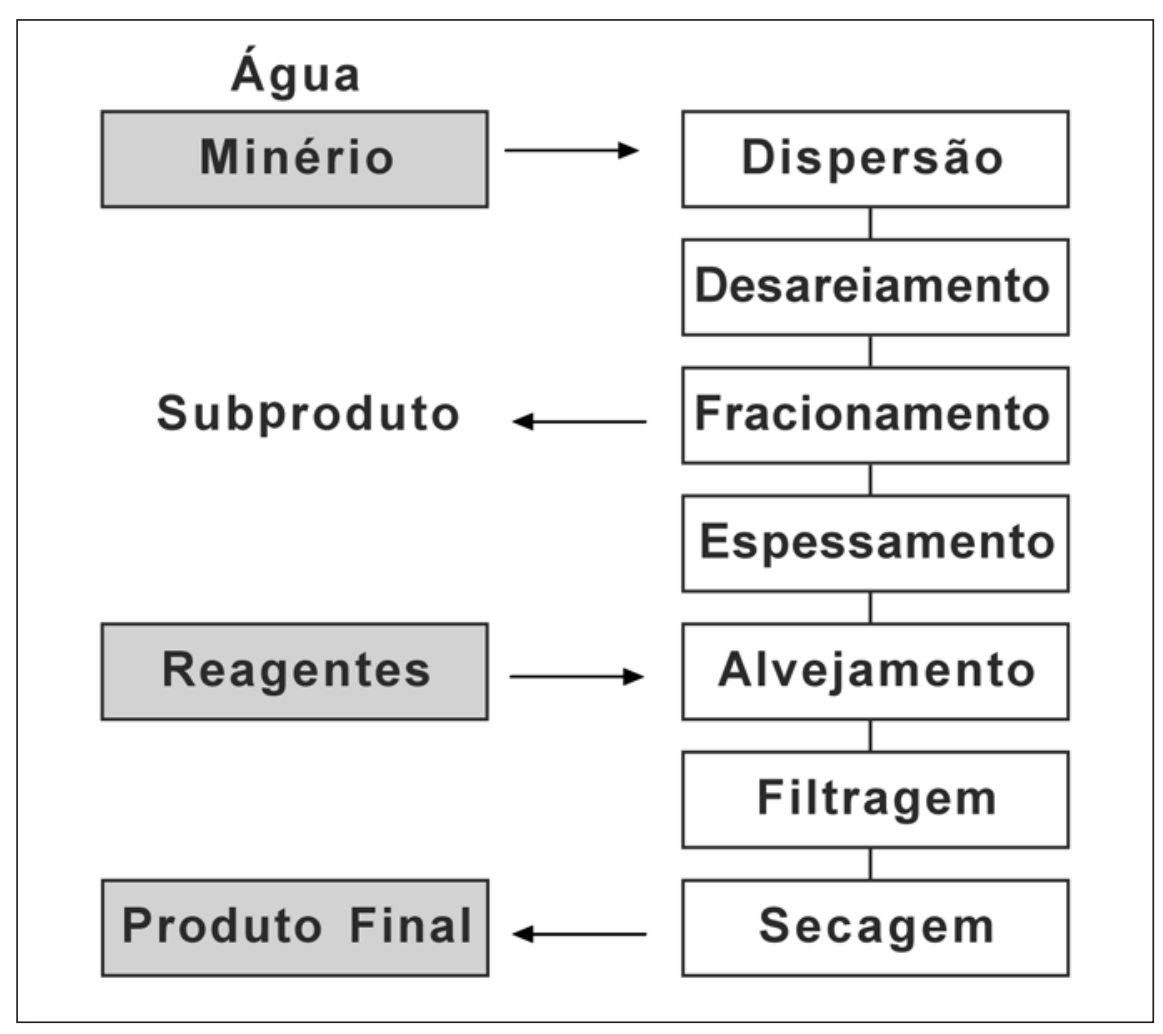

Figura 1 - Fluxograma generalizado do processamento do caulim. 
James José Varela et al.

O CIEL*a*b* é representado por 3 coordenadas ( $L^{*}, a^{*}$ e b*), que são obtidas por meio de transformações matemáticas dos valores tristimulus $\mathrm{X}, \mathrm{Y}$ e Z onde :

- $\mathrm{L}^{*}$ mede a luminosidade, que varia de zero (para o preto) a 100 (para o branco nominal).

- a* varia de positivo a negativo: quanto mais positivo, mais vermelha é a cor e quanto mais negativo, mais verde é a cor da amostra.

- $b^{*}$ também varia de positivo a negativo: quanto mais positivo, mais amarela é a cor e quanto mais negativo, mais azul é a cor da amostra.

As diferenças de cor $\Delta \mathrm{E}(\mathrm{DE})$ para esse sistema são calculadas a partir das diferenças dos valores Lab* entre amostras, utilizando-se a equação 1 :

$$
\Delta E=\sqrt{\Delta L^{* 2}+\Delta a^{* 2}+\Delta b^{* 2}}
$$

Também é muito comum a utilização de índices colorimétricos nas indústrias. Os índices colorimétricos mais utilizados no controle de qualidade de minerais industriais são os índices de alvura e amarelamento (Gliese \& Petter, 2001). Esses índices são calculados a partir dos valores de reflectância da amostra.
A determinação dos índices colorimétricos apresentados nesse trabalho está fundamentada nos procedimentos definidos pelas normas ISO 2470 e ASTM E313-00. Esse método tradicional de caracterização colorimétrica é realizado em pastilhas confeccionadas após secagem, pulverização e prensagem da amostra.

Todos os valores colorimétricos apresentados nesse trabalho foram determinados através de um espectrofotômetro portátil, marca Minolta e modelo CM-508d, com SCE (Componente Especular Exclusa). Esse equipamento foi utilizado, tanto para a aquisição dos valores colorimétricos pelo método tradicional (via pastilha seca prensada), quanto pelo sistema Colorcell (amostras em polpa).

A tecnologia Colorcell, utilizada nesse trabalho, também está em fase de pesquisa para o controle de outros processos contínuos, como, por exemplo, a produção de celulose e papel. A idéia básica empregada no equipamento Colorcell é a passagem da amostra líquida por uma célula de medição, onde a medida de reflexão realizada por um espectrofotômetro é obtida por cima de uma lente projetada para tal propósito (Varela, 2002).

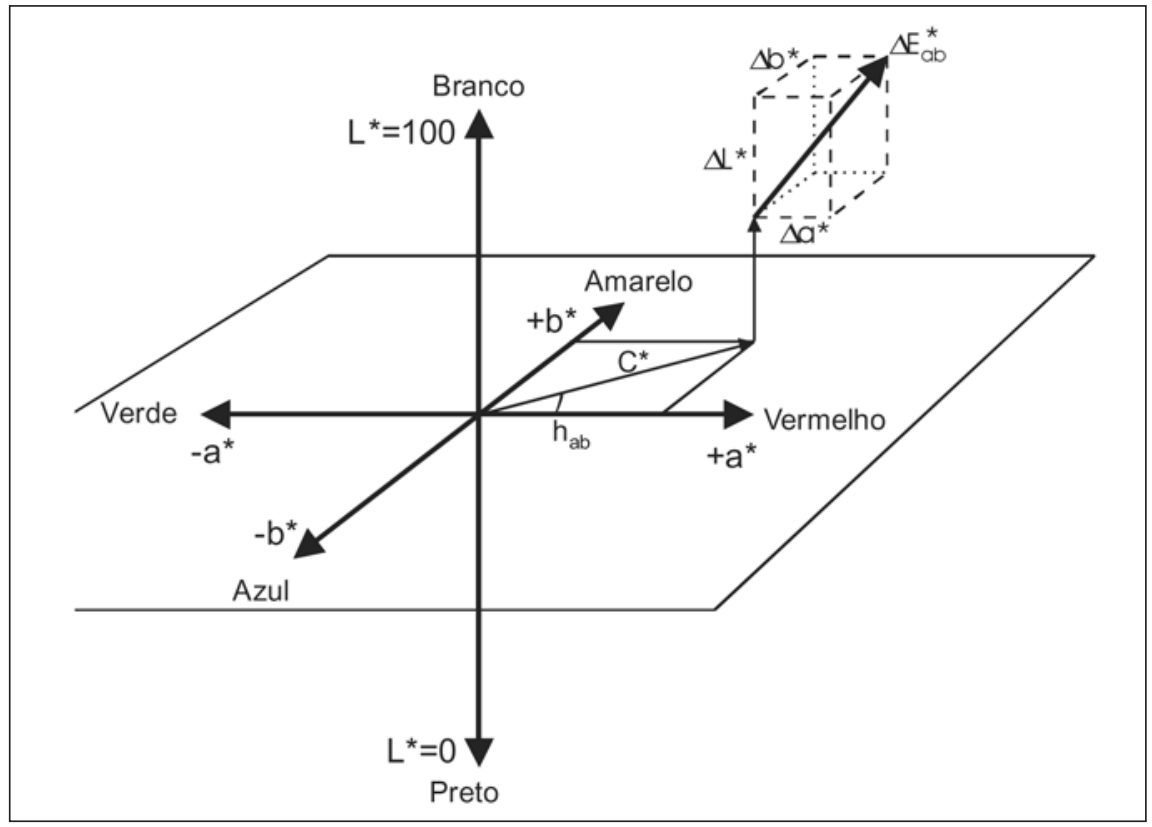

Figura 2 - Representação do sistema colorimétrico CIEL*a*b*, 1976 (Minolta, 1998)
O sistema Colorcell, ver Figura 3, instalado no LAPROM/UFRGS, consiste em: um tanque de aço inox com capacidade máxima de 5 litros e um agitador para homogeneização da polpa (A); uma bomba de polpa com rotação controlável (B); uma célula de medição com lente de quartzo (C); um espectrofotômetro portátil (D); quatro válvulas para limpeza do sistema (E); mangueiras para a recirculação da amostra (F) e limpeza do sistema (G); um painel de controle (H) e um computador conectado ao espectrofotômetro (opcional) (I).

\subsection{Ensaios de alvejamento químico}

Foram realizados três ensaios de alvejamento com a amostra de polpa de caulim coletada na alimentação da etapa de alvejamento da empresa. Nesses ensaios de alvejamento realizados no tanque do sistema Colorcell, utilizaram-se o mesmo agente redutor do ferro (ditionito ou hidrossulfito de sódio) e a mesma quantidade empregada pela empresa no dia amostrado ( $2 \mathrm{Kg} /$ ton). O pH da reação, nos três ensaios, foi ajustado com diferentes quantidades de ácido (mantendo sempre a relação entre os ácidos fosfórico/sulfúrico igual a 2,5).

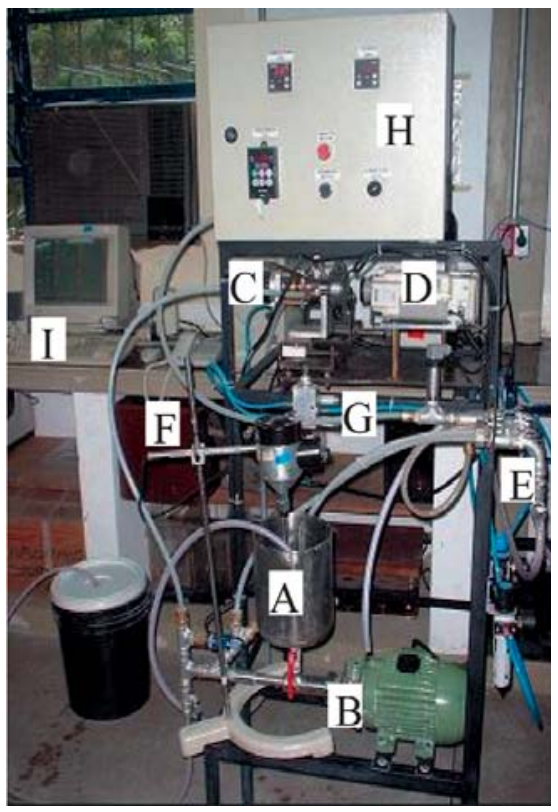

Figura 3 - Sistema Colorcell. 
Controle de qualidade no processamento de polpas de caulim utilizando propriedades óticas

\section{Resultados e discussão}

$\mathrm{O}$ produto alvejado da planta industrial (amostra 2) apresentou um $\mathrm{pH}$ final de 3,2 e os valores de $\mathrm{pH}$ final dos ensaios de alvejamento em laboratório (amostra I, II, III) foram, respectivamente, 3,7; 3,3 e 3,2. As Tabelas 1 e 2 e as Figuras 4 e 5 apresentam os resultados colorimétricos das amostras de caulim alvejadas.

Os parâmetros colorimétricos a* das amostras I, II e III, determinados através do método tradicional, são maiores do que o valor obtido para a amostra 2 .
Esse resultado revela que a amostra alvejada industrialmente possui uma coloração menos avermelhada do que a amostra alvejada em laboratório.

Os resultados apresentados na Tabela 2 confirmam os resultados observados através do método tradicional. Esse fato indica que os alvejamentos de laboratório resultam em produtos com uma qualidade inferior aos produtos obtidos na indústria.

As Figuras 4 e 5 apresentam os valores de reflectância em função do comprimento de onda da luz para as amostras 1 e 2 coletadas na planta de benefi- ciamento. Nas mesmas figuras, encontram-se as curvas espectrais para as amostras I, II e III, que representam os alvejamentos realizados em laboratório (no tanque do sistema Colorcell). Visualizam-se os menores valores, para a amostra 1, e os maiores valores, para a amostra 2, principalmente na início da região visível do espectro eletromagnético (comprimentos de onda próximos a 400 $\mathrm{nm})$.

Os índices de alvura das amostras, que são calculados a partir dos espectros de reflectância, estão apresentados na Tabela 3. Nessa tabela, pode-se visualizar a diferença, em termos de alvura,

Tabela 1 - Diferenças de cor entre o alvejamento industrial e os ensaios em laboratório - método tradicional.

\begin{tabular}{c|c|c|c|c|c|c|c|c|c}
\hline Amostra & $\mathbf{L}^{*}$ & $\mathbf{a}^{*}$ & $\mathbf{b}^{*}$ & $\mathbf{D L}^{*}$ & $\mathbf{D a}^{*}$ & $\mathbf{D b}^{*}$ & $\mathbf{D E} \mathbf{E}^{*} \mathbf{b}$ & Alvura & Amarelamento \\
\hline $\mathbf{2}$ & 96,2 & $-0,7$ & 4,2 & - & - & - & - & 85,0 & 7,5 \\
\hline $\mathbf{I}$ & 95,4 & 0 & 6,1 & 0,7 & $-0,7$ & $-1,9$ & 2,2 & 80,7 & 11,6 \\
\hline II & 95,0 & 0,5 & 6,5 & 1,2 & $-1,2$ & $-2,3$ & 2,9 & 79,3 & 12,8 \\
\hline III & 94,0 & 1,5 & 7,2 & 2,2 & $-2,2$ & $-3,0$ & 4,3 & 76,2 & 14,9 \\
\hline
\end{tabular}

Tabela 2 - Diferenças de cor entre o alvejamento industrial e os ensaios preliminares em laboratório - sistema Colorcell.

\begin{tabular}{c|c|c|c|c|c|c|c|c|c}
\hline Amostra & $\mathbf{L}^{*}$ & $\mathbf{a}^{*}$ & $\mathbf{b}^{*}$ & $\mathbf{D L}$ & $\mathbf{D a}$ & $\mathbf{D} \mathbf{b}^{*}$ & $\mathbf{D E}$ ab & Alvura & Amarelamento \\
\hline $\mathbf{2}$ & 78,9 & $-1,6$ & 8,3 & - & - & - & - & 47,2 & 16,6 \\
\hline I & 76,3 & $-0,5$ & 11,1 & 2,6 & $-1,1$ & $-2,8$ & 4,0 & 40,9 & 23,9 \\
\hline II & 75,8 & 1,1 & 12,5 & 3,1 & $-2,7$ & $-4,2$ & 5,9 & 39,1 & 28,4 \\
\hline III & 74,0 & 3,4 & 14,3 & 4,9 & $-5,0$ & $-6,0$ & 9,2 & 35,2 & 34,8 \\
\hline
\end{tabular}

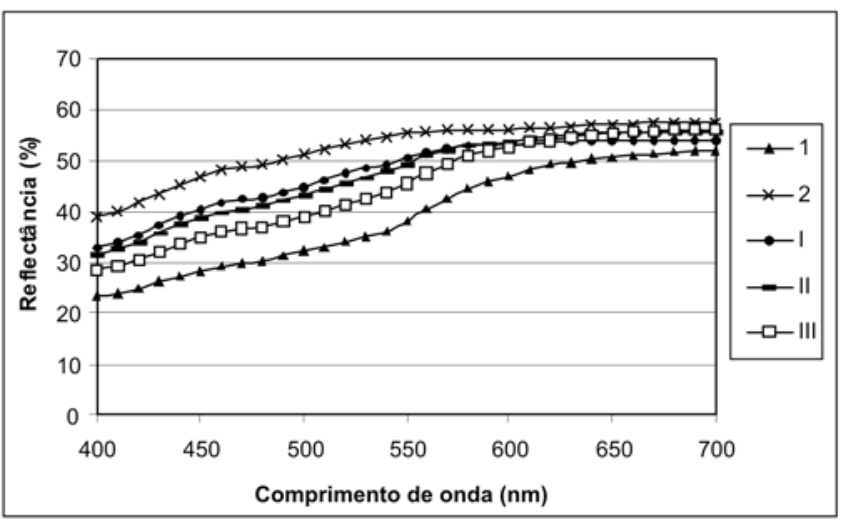

Figura 4 - Espectros de reflectância das amostras de caulim alvejadas - método tradicional.

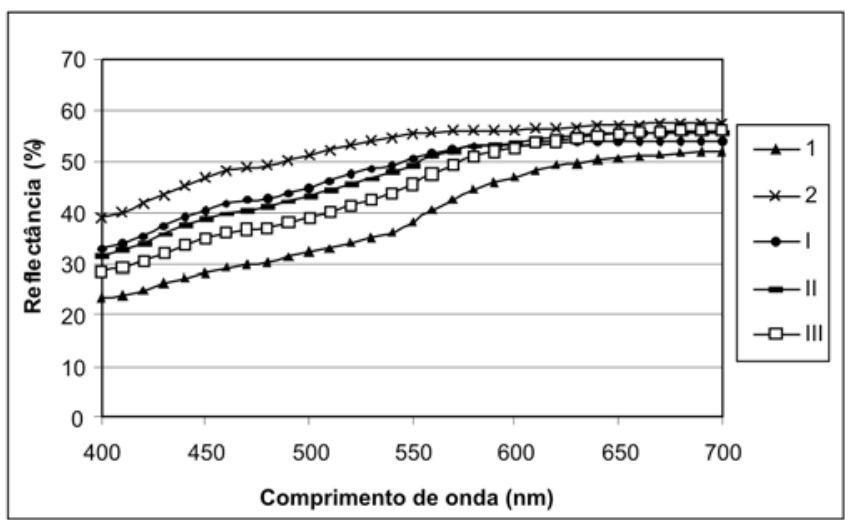

Figura 5 - Espectros de reflectância das amostras de caulim alvejadas - sistema Colorcell. 
entre os produtos dos distintos alvejamentos. A amostra seca de caulim, antes de ser alvejada, caracterizada pelo método tradicional, apresentou um índice de alvura de $70,2 \%$, enquanto que a amostra em polpa, caracterizada com o sistema Colorcell, apresentou um valor de alvura de $28,7 \%$.

Os índices de alvura obtidos com os ensaios preliminares de alvejamento revelam a importância do $\mathrm{pH}$ da polpa na etapa de alvejamento. Comparando-se os produtos obtidos em laboratório (amostras I, II e III), observou-se que o alvejamento realizado com um $\mathrm{pH}$ maior $(\mathrm{pH}=$ $3,7)$ resultou em um produto de melhor qualidade, em termos de alvura, do que o alvejamento realizado com um $\mathrm{pH}$ menor $(\mathrm{pH}=3,3$ e 3,2$)$.

Utilizando-se como critério de comparação o valor de $\mathrm{pH}$ final da reação, pode-se avaliar a diferença de qualidade dos caulins alvejados. Verifica-se, na tabela 1 , que a diferença de cor $(\Delta \mathrm{E})$ avaliada pelo método tradicional entre o alvejamento industrial e o de laboratório (ambos com pH final igual a 3,2) foi da ordem de 4,3 pontos.

A diferença entre o alvejamento em laboratório (amostra III) e o industrial (amostra 2), em termos de alvura úmida, representa 12 pontos. Esse mesmo valor, em termos de alvura seca, representa 8,7 pontos. A eficiência do alvejamento da amostra III, em relação à amostra 2 , para a alvura seca, é de aproximadamente $90 \%$; em termos de alvura úmida, a eficiência é de $75 \%$.
Existem alguns fatores que, possivelmente, poderiam explicar a diferença dos produtos obtidos entre a escala industrial e a de laboratório, como, por exemplo:

- Reagentes: diluição dos ácidos, oxidação do hidrossulfito. Luz e Chaves (2000) ressaltam o cuidado que se deve ter com a decomposição do hidrossulfito, encontrado comercialmente sob a forma de pó cristalizado $90 \% \mathrm{Na}_{2} \mathrm{~S}_{2} \mathrm{O}_{4}$, em tiossulfato e bissulfito (equação 2). Para se evitar esse efeito indesejável, é recomendável mantê-lo abaixo de $10^{\circ} \mathrm{C}$.

$2 \mathrm{~S}_{2} \mathrm{O}_{4}^{-2}+\mathrm{H}_{2} \mathrm{O} \Leftrightarrow \mathrm{S}_{2} \mathrm{O}_{3}^{-2}+2 \mathrm{HSO}_{3}^{-}$

- Agitação e homogeneização da polpa nos tanques de alvejamento: além do fator de escala, relacionado aos ensaios, encontram-se dados na literatura (Luz e Chaves, 2000) apontando para o fato de que a perda do reagente hidrossulfito também pode ser causada por uma reação de oxidação para sulfito e sulfato na presença de oxigênio. Esse efeito é atenuado na etapa de alvejamento utilizando baixas velocidades de agitação (<100 rpm), para que não entre ar para a suspensão.

- Recirculação da polpa no sistema Colorcell.

- Tempo de análise: a medição a úmido é instantânea; por outro lado, na medição a seco, a amostra passa por etapas de preparação anteriores à caracterização propriamente dita. Esse processamento é muitas vezes de- morado e pode ocasionar alterações no material.

A quantificação das influências que possivelmente, estariam ocasionando uma diferença na qualidade dos produtos alvejados não foi objeto desse trabalho.

A correlação dos índices colorimétricos (alvura e amarelamento) das amostras de caulim, determinados através do método tradicional e através do sistema Colorcell, está apresentada nas Figuras 6 e 7. Considerando que os coeficientes de correlação para as curvas geradas foram superiores a 0,985 , a correlação linear obtida foi considerada satisfatória.

\section{Conclusões}

Uma das principais vantagens proporcionadas pela tecnologia Colorcell é a verificação dos parâmetros de qualidade dos produtos em tempo real.

Os produtos secos de caulim alvejados, caracterizados pelo método tradicional, apresentaram índices de alvura entre 76 a $85 \%$. Enquanto que a amostra em polpa, caracterizada com o sistema Colorcell, apresentou valores de alvura entre 35 a 47\%. Os maiores valores de alvura dos produtos alvejados foram obtidos com os maiores valores de $\mathrm{pH}$ $(3,7)$.

Verificou-se a possibilidade de correlacionar os parâmetros colorimétricos medidos através do método tradicional

Tabela 3 - Comparação dos índices de alvura das amostras alvejadas.

\begin{tabular}{c|c|c|c|c|c|c}
\hline \multirow{2}{*}{ Amostra } & \multicolumn{2}{|c|}{ Alvura } & \multicolumn{2}{c|}{ Diferença * $^{*}$} & \multicolumn{2}{c}{ Eficiência ** } \\
\cline { 2 - 7 } & Polpa & Seca & Polpa & Seca & Polpa & Seca \\
\hline $\mathbf{2}$ & 47,24 & 84,96 & - & - & - & - \\
\hline I & 40,85 & 80,72 & $-6,39$ & $-4,23$ & $86 \%$ & $95 \%$ \\
\hline II & 39,06 & 79,25 & $-8,18$ & $-5,71$ & $83 \%$ & $93 \%$ \\
\hline III & 35,24 & 76,23 & $-12,00$ & $-8,73$ & $75 \%$ & $90 \%$ \\
\hline
\end{tabular}

* Diferença = diferença do valor de alvura da amostra x e amostra 2.

** Eficiência = razão entre o valor de alvura da amostra $\mathrm{x}$ e amostra 2. 
Controle de qualidade no processamento de polpas de caulim utilizando propriedades óticas

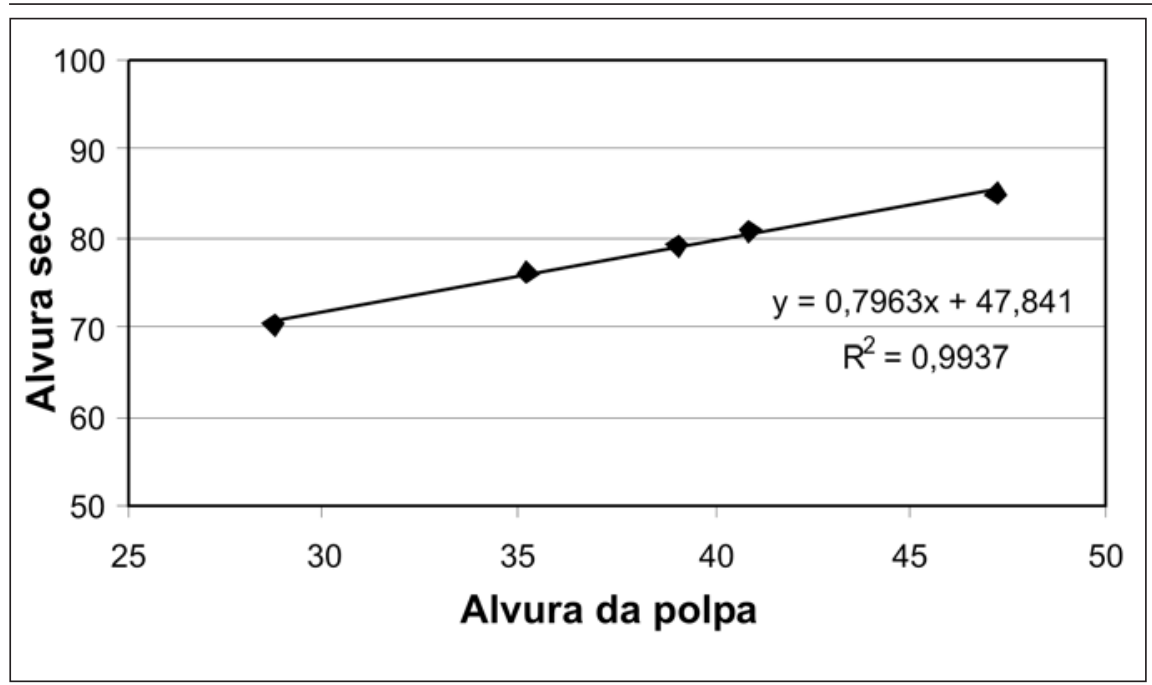

Figura 6 - Correlação entre os valores de alvura seca e úmida das amostras de caulim.

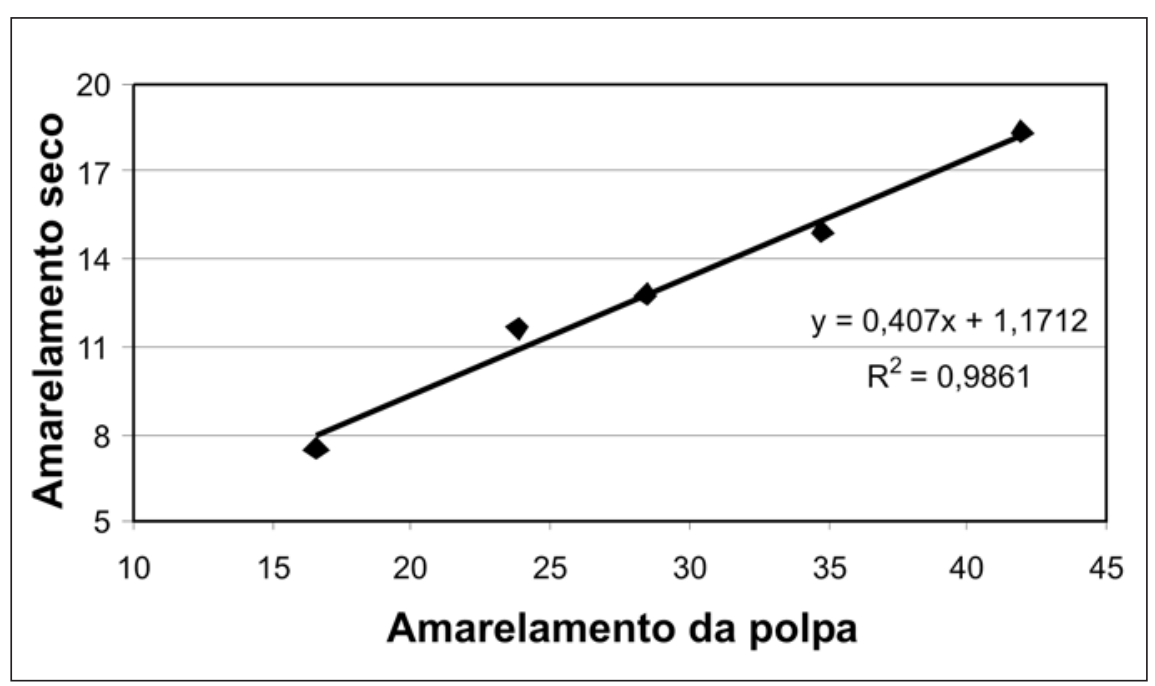

Figura 7 - Correlação entre os valores de amarelamento seco e úmido das amostras de caulim.

e através do sistema Colorcell. Os testes realizados com o sistema Colorcell indicam a possibilidade de se otimizarem as variáveis da etapa de alvejamento (reagentes, tempo de processamento) e, conseqüentemente, reduzirem-se os custos de produção.

\section{Agradecimentos}

Os autores agradecem às Instituições de fomento à pesquisa e desenvolvimento de recursos humanos do Brasil e, também, aos colegas de trabalho da UFRGS pelo apoio recebido.

\section{Referências bibliográficas}

ASTM E313-00 Standard practice for calculating yellowness and whiteness indices from instrumentally measured color coordinates. Norma técnica, 2000. 5p.

CARVALHO, E. A. Caulim e carbonato de cálcio: competição na indústria de papel. Rio de Janeiro: CETEM/CNPq, 1997. 28p. (Série Estudos e Documentos).

GLIESE, R., PETTER, C.O. Parâmetros colorimétricos relevantes para a indústria mineral. In: ENCONTRO NACIONAL DE TRATAMENTO DE MINÉRIOS E METALURGIA EXTRATIVA, 2001. Anais... Rio de Janeiro: XVIII ENTMME, 2001. v. 3, p. 18-22.

ISO 2470 Paper and Board - Measurement of Diffuse Blue Reflectance Factor (ISO Brightness). Norma técnica, 1999. 5p.

LARROYD, F. Geologia e caracterização tecnológica dos principais depósitos de caolim do Estado do Rio Grande do Sul: ênfase à utilização na indústria do papel. Dissertação do Programa de PósGraduação em Engenharia de Minas, Metalúrgica e de Materiais-PPGEM/ UFRGS, 1996. 130p.

LUZ, A.B., CHAVES A.P. Tecnologia do caulim: ênfase na indústria de papel. CETEM/CNPq, 2000. 72p. (Série Rochas e Minerais Industriais).

MINOLTA. Precise color communication color control from perception to instrumentation. Japan: Minolta Co., Ltd., 1998. 59p.

SILVA, L. A. Caracterização tecnológica e beneficiamento de caulins da região de Pântano Grande-RS. Dissertação do Programa de Pós-Graduação em Engenharia de Minas, Metalúrgica e de Materiais-PPGEM/UFRGS, 2000. 103p.

VARELA, J.J. Caracterização de polpas de minerais industriais através do equipamento Colorcell visando ao controle de qualidade do produto. Dissertação do Programa de PósGraduação em Engenharia de Minas, Metalúrgica e de Materiais-PPGEM/ UFRGS, 2002. 181p.

Artigo recebido em 15/09/2003 e aprovado em 04/07/2005.

\section{Conheça o novo site da REM: WWW.rem.com.br}

